\title{
Reliability Analysis of the Internet of Things Based on Ordered Binary Decision Diagram
}

\author{
https://doi.org/10.3991/ijoe.v14i08.9185 \\ Wei Guan \\ Guangdong Polytechnic of Water Resources and Electric Engineering, Guangzhou, China \\ guanwei1973@21cn.com
}

\begin{abstract}
The reliability of the Internet of Things (IoT) system is analyzed and studied through ordered binary decision diagram (OBDD) to improve its design, application, and development. Based on the OBDD analysis, a reliability evaluation method named as enhanced node expansion (ENE) is proposed. This method provides an effective solution for the reliability assessment of IoT with large scale and complex network structure. A link importance assessment method based on OBDD analysis is also established. The proposed method can accurately and effectively evaluate the reliability of the IoT network and is practical for discussing the reliability, design, and development of this system.
\end{abstract}

Keywords-Internet of things, ordered binary decision diagram, reliability, enhanced node expansion method

\section{Introduction}

In 2005, the concept of "the Internet of Things (IoT)" was formally proposed by the International Telecommunications Union (ITU). ITU pointed out in the ITU Internet Report 2005: the Internet of Things that the 21st Century is an omnipresent "Internet of things communication era when all objects from cars to roads, furniture to houses and even the world can communicate with each other according to their needs through the network [1]. At the end of 2008, the chief executive of International Business Machine first proposed the concept of "intelligent earth", namely, embedding sensors in every place around the world and adding labels to all items including cars, clothing, and toothbrushes. These objects are generally connected to form the IoT, and their management, adjustment, and integration are achieved through largescale servers and "cloud computing." Ultimately, "Internet + networking = the intelligent earth" is formed, and IoT is predicted to be the next wave for the information industry [2].

IoT has recently made great progress and has been widely used in many industries, such as, food safety, public safety, health monitoring, intelligent transportation, security, and environmental protection. The network scale is expanding from laboratories to buildings and then to communities, with a convergence of different systems. With the expansion of the network scale, many problems of IoT are exposed: the work environment of IoT is complex and mainly uses the wireless way to transmit the sig- 
nal, the stability of the communication link is deficient, the terminal of IOT is widely distributed, and the structure is simpler than the terminal of the computer network and thus faces the shortcomings of the terminal work deficiency and vulnerability to attack. The network is a mobile network without a fixed topology, and the overall connectivity of the network is not guaranteed. The applied industries of IoT, such as food safety and intrusion detection, require that IoT provides rapid and accurate response to unexpected events, users, and managers and realizes the accurate communication between people and things. The infrastructure of the network must also have an economic department. This network requires coordinated operation of the system in an efficient and reliable mode, and reliability is fully considered in the network design, deployment, and network management phases.

\section{State of the art}

Ordered binary decision diagram (OBDD) is a standard expression form of Boolean function and a new data structure. Based on OBDD, Boolean functions can be effectively expressed and operated, which can be regarded as a new abstract data type. The successful application of OBDD in large-scale integrated circuit logic synthesis and verification has attracted attention from academia and industrial application circles. To date, OBDD technology and its industrial application have made considerable progress and produced considerable research results.

Bernasconi studied the fault tolerance of OBDD and designed an elastic version of the data structure, that is, self-repair OBDD, which makes the reduced OBDD elastic to errors in the indexes. The indexes are related to the input variables or in the pointer of the node. These strategies take advantage of the inherent redundancy of data structures and efficiently implement the redundant introduced [1]. Ali et al. proposed a decision support framework for air traffic management rescheduling based on ground delays, changing initial flights, and flight cancellations. This method is based on the use of time Petri net (TPN) to simulate the air traffic network. A state space representation method of time Petri net based on binary decision diagram is introduced. This tool, known as the time reduced ordered binary decision diagram (T-ROBDD), can represent a large state space of TPN with a small data structure, and can effectively operate the set [2]. Li et al. changed the access structure of access strategies described in cipher-text policy attribute-based encryption (CP-ABE) and proposed a $\mathrm{CP}-\mathrm{ABE}$ system based on ordered binary decision diagram (OBDD). The new system takes full advantage of OBDD's powerful descriptive ability and high computational efficiency [3]. Bollig believed that for a given function, the size and width of the OBDD are very sensitive to the selection of the variable sort, but the calculation of the optimal variable ordering for the OBDD size is called NP-hard. The optimal variable ranking relative to the OBDD size is not necessarily optimal for the complete model or the OBDD width, and the relationship between the optimal variable ordering about the size and width is almost unknown. He explored this relationship, used a new simplified idea, and showed that the complete OBDDS size and width minimization problem is NP-hard's [4]. For tight class results of function classes represented by K layers 
of ordered binary decision diagrams determined by polynomial size, Brosenne et al. studied uncertainty, where the layer was driven by the same variable. $\mathrm{K}$ is a constant that shows that a similar hierarchy collapses for existence, parity, and most accepted modes [5]. Bibilo presented a multilevel representation algorithm for incomplete definitions of Boolean function systems with various initial functions. Multilevel representation is based on Shannon decomposition. The experimental results show that the proposed algorithm is effective for the synthesis of library element logic circuits [6]. Marugán et al. believed that enterprises needed to optimize any decision made to improve their competitiveness. The relationship between reasons and decisions is influenced by the decision-making process, and the best solution for each logistical or organizational problem could be established by finding the main reasons involving the best decisions. The decision process is analyzed qualitatively and quantitatively using the logical decision tree and binary decision diagram. Moreover, a new kind of different ranking method is proposed for the logical decision tree of several case studies [7]. Khadiev discussed the famous K-OBDD model and proved the breadth-based hierarchy of Boolean function classes based on K-OBDD. The proof of the hierarchy is the sufficient condition that Boolean functions are represented as the complex properties of K-OBDD and Boolean function-server application function. The function is the modification of the known pointer jumping (PJ) and indirect storage access (ISA) function [8].

Most scholars studied the theory and calculation method of OBDD; however, only a few focused on its application to the reliability of the IoT. Therefore, in view of the above situation, this paper analyzes and studies the reliability of the IoT by using ordered binary decision diagram.

\section{$3 \quad$ Method}

\subsection{Analysis of the architecture of the IoT}

The IOT is a huge information system, covering all aspects of information technology, including, coding, sensing, communication, network, and many other aspects, with a variety of architectures. The structure of the IoT proposed by China mainly includes three parts shown in Figure 1.

The bottom is the perceptual layer, which mainly realizes the object information acquisition through the sensor and ratio frequency identification (RFID) electronic tags. It also transforms the features of the objects into data that can be transferred and processed. The middle is the network layer, mainly including the Internet (IPV4 and IPV6), mobile network ( $2 \mathrm{G}, 3 \mathrm{G}$, and $4 \mathrm{G})$, and radio and television network, to realize the long-distance transmission of information. The upper is the application layer, which realizes the specific intelligent application and completes the information exchange between objects and between people and objects. The IoT can be understood as a data collection link added on the basis of the existing Internet, which transfers the data collected by the sensor, the information of the electronic label and all kinds of other information to the existing network to realize the interaction of the information. 


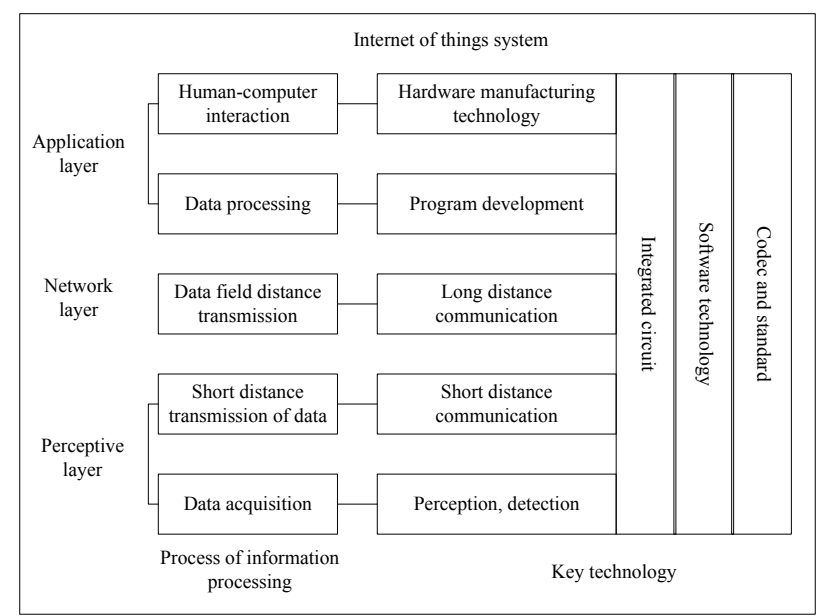

Fig. 1. Typical architecture of the IoT

The perception layer is the end, collecting object information in the IoT. This layer is located at the bottom of the three-layer structure of the IoT, which digitizes the physical characteristics of the objects. Therefore, the perception layer is the foundation of the whole industrial chain and the link with the largest total demand. The most important technology for the application of the perception layer is RFID. RFID refers to the use of radio frequency signals to achieve non-contact transmission. The system is usually composed of an electronic tag, a reader and an upper unit, as shown in Figure 2. The workflow of the RFID system is that the reader sends the encoded information around the transmitter through the transmitting antenna. When the label enters the working area of the transmitting antenna, it receives the signal and activates the label. The activated tag determines the reader's request and permission information according to the received information and completes the corresponding operation according to the judgment result. If the outcome is a read command, then the tag sends the corresponding information to the reader through the antenna, and the reader receives the data and sends them to the upper unit main system for related processing. If the outcome is the written command, then the tag modifies the internal data in accordance with the reader's request.

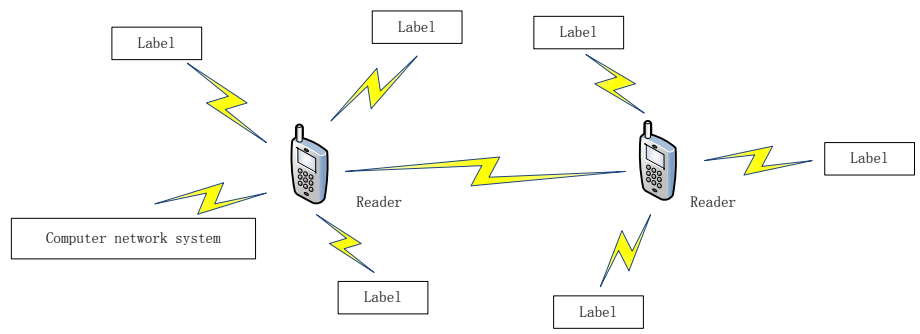

Fig. 2. Basic composition of RFID system 
The network layer is located in the middle of the three-layer structure of the IoT and mainly connects the perception layer to the traditional network, the user access, and the communication of information according to the application layer. The main technologies include wireless ad hoc networks and traditional wired and wireless networks. Wireless ad hoc networks (AdHoc) can independently build networks without the support of fixed network facilities. All nodes in the network have equal status, no special central control node exists, and the nodes can communicate directly with those in their own communication range. When the distance between the destination node and the source node exceeds the direct communication distance of the node, they can communicate through the intermediate node forwarding data, that is, the data must pass through more jumps so that they can reach the destination. This communication requires that nodes in the network not only have the functions required by ordinary mobile terminals, but also have the routing function of message forwarding. AdHoc network is not dependent on fixed and special network facilities. This system can quickly establish and close the network and can also access Internet or cellular wireless network. This feature provides a favorable guarantee for its extensive use in the IoT.

The application layer is the interface between the IoT and users (people, organizations, and other systems), which is combined with the specific needs of the industry to realize the diversified application of the IoT. The key technologies include complex data management and open-loop management of different application systems.

\subsection{Reliability analysis}

Reliability theory had originated in the early 1920s. The earliest research area was machine maintenance, but it did not receive enough attention. During the Second World War, a large number of war equipment and equipment encountered the large scale of scrap in the process of transportation and storage due to low reliability. As a result, countries began to pay attention to reliability research, which has become the focus of many researchers. With the development of science and technology, all kinds of systems become increasingly large and complex, and the working environment diversifies. This outcome increases system requirements to not only accuracy but also high reliability.

The general definition of reliability given by C.Ebelingtl is that reliability refers to the probability that the product completes the specified function within a given time under the prescribed operating conditions. The product contains everything from the system, equipment to components. Specified conditions refer to all external conditions of a product when it is in operation, including, natural environment, use, maintenance, and other conditions. The prescribed function refers to the performance technical index of the product. In general, with the improvement of the performance index and technical requirement, the possibility of the product's completion of the specified function will be reduced. This definition defines the three basic elements of reliability, including conditions, time, and functions. Reliability is a kind of probability that varies with time where the product does not fail. In many cases, reliability 
degree is used to measure the probability of reliability, expressed as $\mathrm{R}(\mathrm{t})$, which indicates the probability of a product working normally in time $t$, as shown in formula (1).

$$
R(t)=P(T>t)=1-F(t)=\int_{0}^{t} f(t) d(t) .
$$

In (1), $T$ indicates the normal working time of the product, and $f(t)$ is the product failure distribution density function.

The large number of terminal devices in the IOT is the basis for realizing the function of the system. Only when the equipment is working normally and without fault can the system function be operated. The reliability of the equipment is characterized by the inherent reliability of the system itself. Many terminals are in the state of unmanned surveillance for a long time. Human interference occurred during the work, which requires the terminal to have high reliability.

Communication in the IoT greatly differs from general communication. The main difference is the large number of nodes in the IoT. Communication is not a single one-to-one communication but a multi-to-one communication. The working environment of the communication node is complex, and the structure of the node is relatively simple, easy to be disturbed and attacked by the outside world. The node in the IoT is dynamic and the communication link is unstable. The reliability of communication in the environment of IoT is analyzed in the light of the situation of electromagnetic interference and the movement of communication nodes.

The network is the core of the IoT. The reliability of the IoT represents the reliability of the system to a certain extent. The reliable connectivity of the network is the main direction of the research of network reliability, but the connectivity of the network cannot fully reflect the reliability of the network. Assessing the reliability of the network from all aspects is necessary, including the network connectivity rate, network capacity, delay, and packet loss rate, which are the main contents of the reliability of the IoT.

\subsection{Reliability evaluation based on ordered binary decision diagram}

Binary decision diagram (BDD) is an efficient structured expression of Boolean functions. BDD can reduce the storage space by classifying the equivalent state of Boolean functions into the same structure. The if-then-else Boolean operator is $\mathrm{x} \rightarrow \mathrm{y}_{0}$ and $\mathrm{y}_{1}$ is $\mathrm{x} \rightarrow \mathrm{y}_{0}, \mathrm{y}_{1}=\left(\mathrm{x} \Lambda \mathrm{y}_{0}\right) \vee\left(-\mathrm{x} \Lambda \mathrm{y}_{1}\right)$. The Boolean expression consisting of only the if-then-else Boolean operator, the Boolean variable, and the Boolean constants 0 and 1 is called INF paradigm. If the expression of Boolean variable $\mathrm{x}$ in the Boolean expression $t$ is marked as $t \mid x=1$ and the expression of $x$ valued 0 is marked as $t \mid x=0$, then it is not difficult to find the equivalent form of the Boolean expression $t$ :

$$
t=\left.x \rightarrow t\right|_{x=1},\left.t\right|_{x=0}
$$

The above formula actually gives a way to transform the Boolean expression $t$ into an equivalent INF paradigm. The similar equivalent transformation of Boolean varia- 
bles in $\mathrm{t}$ is performed one by one, the process is represented by a tree graph then the binary decision diagram (BDD) can be obtained.

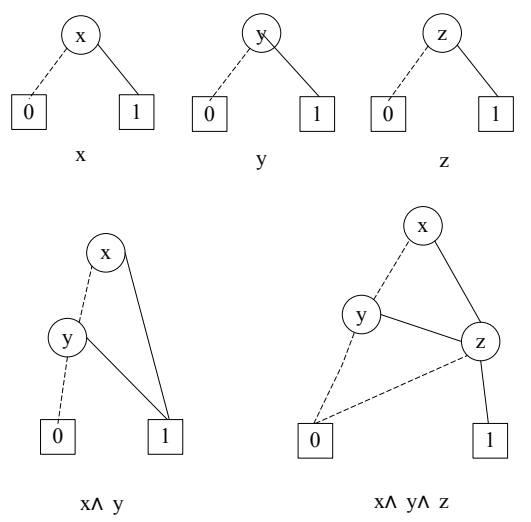

Fig. 3. BDD expression of Boolean function

In Figure 3, the Boolean function $\mathrm{f}=\mathrm{x} \vee \mathrm{y} \wedge \mathrm{z}$ is taken as an example to illustrate the structure form of BDD. BDD has a hierarchical feature, which consists of multi-layer circular nodes and the bottom two rectangular nodes. Each layer can have multiple circular nodes, but all round nodes in the same layer except the lowest layer correspond to the same Boolean variables; the lowest two nodes are rectangular, representing logical true or logical false. Each circular node has two directional edges to point to the next layer. One edge is marked with a solid line and the other, a dotted line: the solid line edge points to the bottom structure when the node takes 1 , also known as the 1- child; the dashed line edge points to the bottom structure when the node takes 0 , called the 0 - child. The same Boolean function can generate different structures but equivalent BDD through different order of variables. Therefore, by optimizing the order of variables, the number of nodes in BDD can be reduced, and the overall computational complexity can be reduced.

If the BDD is created for a Boolean function in a clear sequence of variables, the result is an ordered binary decision diagram, namely, an ordered BDD. Breadth First Search (BFS) has been proved to be an effective OBDD ranking principle, and this BFS principle is also adopted here. From the above analysis and method, any Boolean function can be transformed into a non-circular ordered binary decision diagram issue.

In the IoT network, a number of node clusters are placed in the area to be monitored, and each node cluster is composed of many sensor nodes. The data monitored by the sensor node will be sent to the Sink end of the data terminal. In data transmission, certain nodes in the cluster serve as intermediate nodes to relay data between other clusters and Sink terminals. As far as monitoring environmental data targets are concerned, the reliability of the IoT network is the probability that there is at least one efficiently working data connected path between the Sink end and the sensor node. According to the description of the undirected graph model in Figure 4, the reliability 
of the IoT can be defined as the connectivity probability between the two nodes in the undirected graph, that is, the reliability of the two terminals networks. The two terminals here refer to the two endpoints of the Sink end and sensor nodes in the IoT. Based on the research methods and results of the two terminals' reliability of the IoT network, this system can be similarly used in the reliability of K-end network between $\mathrm{K}$ endpoints and the full-end network reliability between all endpoints in the network.

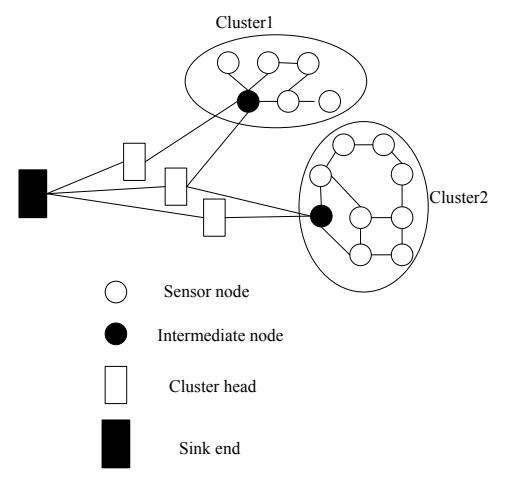

Fig. 4. Undirected graph representation of Internet of things

According to the above analysis, the IoT can be represented by an undirected random graph $\mathrm{G}=(\mathrm{V}, \mathrm{E})$, where $\mathrm{V}$ is a set of nodes and $E \subseteq V \times V$ is a set of edges. For each node $v_{i}$, the probability of normal work is $p_{i}$, and the probability of failure of the node is $\left(1-p_{i}\right)$. The edge between the node $v_{i}$ and node $v_{j}$ is written as $v_{i j}$, whose probability is $p_{i j}$, and the probability of failure at that edge is $\left(1-p_{i j}\right)$. The undirected random graph $\mathrm{G}=(\mathrm{V}, \mathrm{E})$ model is applied to study the two-terminal reliability of the IoT, based on the following two assumptions. One is to assume that the failure of nodes and edges is statistically independent. The second one is to assume that the topological structure remains the same.

The reliability of graph $\mathrm{G}=(\mathrm{V}, \mathrm{E})$ can be represented by the following Boolean expression:

$$
f(G)=\mathrm{V}_{v\{\text { adjacentnodeofs }\}}\left(\left(x_{0}\right) \wedge f\left(G_{s=>v}\right)\right) .
$$

In (3), $\mathrm{s}$ is the source node, $\mathrm{v}$ is the node adjacent to $\mathrm{s}, \mathrm{x}_{0}$ is the Boolean variable of the node, and $G_{s=>v}$ is the subnet of the network $\mathrm{G}$ after the source node s expands to the node v. According to this expression, the construction function of OBDD of network $\mathrm{G}$ can be constructed recursively by the following four steps. The first step is to determine whether the current source node is adjacent to the final target node. If it is adjacent, it returns to bddtrue; if the current source node has no node adjacent to it, it returns bddfalse; otherwise, it will continue the next step. The second step is to remove useless redundant nodes to avoid invalid operations. In addition to the source node and the final target node, if a node has only one edge connected to it, then the node is a redundant node. The third step is to check and compare the subnet hash table 
to see if a subnet OBDD that is isomorphic to the current subnet exists in the hash table. If an isomorphism sub OBDD exists in the hash table, then it will return to isomorphic subnet OBDD. If no isomorphic subnet OBDD exists, then proceed to the next step. This discriminating step can avoid repeated construction of isomorphic subnet OBDD and save considerable computation time. The fourth step is to perform the Boolean operations on the OBDD and store the result robdd $(\mathrm{G})$ in the hash table.

$$
\begin{aligned}
& r_{\text {obdd }}\left(G^{\prime}\right)=r_{\text {obdd }}(x j) \text { and } \quad r_{\text {obdd }}\left(G_{s=>v j}\right) ; \\
& r_{\text {obdd }}(G)=r_{\text {obdd }}(G) \text { or } \quad r_{\text {obdd }}\left(G^{\prime}\right) .
\end{aligned}
$$

As an example, Figure 5 is the OBDD structure of the network $G$ built by the ENE method, in which $\mathrm{v}_{0}$ is the source starting point, $\mathrm{v}_{5}$ is the target node, and $\mathrm{f}=\mathrm{x}_{0} \Lambda\left(\left(\mathrm{x}_{1} \Lambda \mathrm{x}_{3}\right) \vee\left(\mathrm{x}_{2} \Lambda \mathrm{x}_{4}\right)\right) \Lambda \mathrm{x}_{5}$.

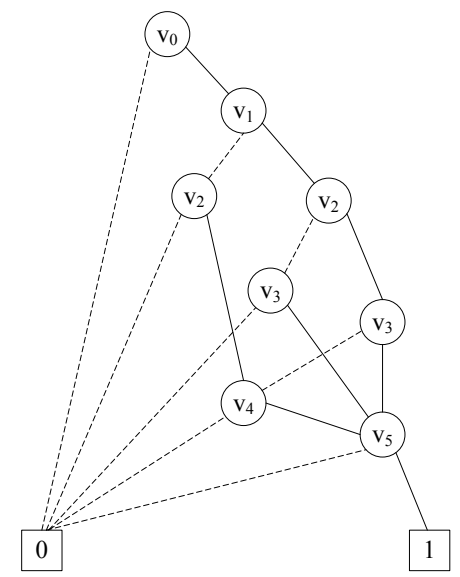

Fig. 5. OBDD structure of network G

According to the Shannon decomposition theorem:

$$
f=b \mathrm{~g} f_{b=1}+\bar{b} g f_{b=0} .
$$

The reliability of the network can be calculated as follows:

$$
\begin{aligned}
& f_{\text {rel }}(G)=f_{\text {rel-obdd }}\left(r_{\text {obdd }}(G)\right)= \\
& \operatorname{Pr}\left(x_{i}=1\right) \times f_{\text {rel-obdd }}\left(\left.r_{\text {obdd }}(G)\right|_{x_{i}=1}\right) \\
& +\operatorname{Pr}\left(x_{i}=0\right) \times f_{\text {rel-obdd }}\left(\left.r_{\text {obdd }}(G)\right|_{x_{i}=0}\right) .
\end{aligned}
$$

In (6), $r_{\text {obdd }}(G)$ indicates the OBDD construction function of the network G, $\left.r_{\text {obdd }}(G)\right|_{x_{i}=1}$ means the OBDD construction function of subnet when $\mathrm{x}_{\mathrm{i}}=1$, and $\left.r_{\text {obdd }}(G)\right|_{x_{i}=0}$ represents the OBDD construction function of subnet when $\mathrm{x}_{\mathrm{i}}=0$. 
The reliability of $\mathrm{G}=(\mathrm{V}, \mathrm{E})$ can be calculated through a series of calculations. The failure factor is not taken into account in the reliability obtained. By variable permutation, the variable displacement is conducted by adding the edge variable for the node variable $\mathrm{x}$ in equation (3). $\left(\mathrm{x}_{\mathrm{j}}\right)$ is replaced to $\left(\mathrm{x}_{\mathrm{ij}}\right) \wedge\left(\mathrm{x}_{\mathrm{j}}\right)$, and the $\mathrm{x}_{\mathrm{ij}}$ is a Boolean variable of the edge $e_{i j}$. The reliability formula (6) is changed accordingly.

$$
\begin{aligned}
& f_{\text {rel }}(G)=\left(p_{i j} \times p_{j}\right) \times f_{\text {rel-obdd }}\left(\left.r_{\text {obdd }}(G)\right|_{x_{j}=1}\right) \\
& +\left(1-p_{i j} \times p_{j}\right) \times f_{\text {rel-obdd }}\left(\left.r_{\text {obdd }}(G)\right|_{x_{j}=0}\right)
\end{aligned}
$$

In (7): $p_{j}$ is the probability of node $v_{j}$ working normally, and $p_{i j}$ is the working probability of edge $e_{i j}$ between node $v_{i}$ and node $v_{j}$. At this point, through the iterative calculation of OBDD structure and reliability, the results of network G two-terminal network reliability calculation can be obtained.

The main operation of the ENE method lies in the recursive call process. Each nested call is the reliability calculation of the subnet of the current IoT network, and the ENE method uses the hash table and the three-level variable long bit vector label method to avoid the repeated calculation of the isomorphic subnet. In addition, the recursion process can be figuratively understood as the non-repeated traversal of the vertex of graph G. Therefore, the two-stage operation OBDD construction of ENE method and the complexity of reliability calculation process are both less than $O(n)$. According to the above analysis, the total complexity of ENE method is less than $\mathrm{O}(2 \mathrm{n})$. If the edge order is optimized, the complexity of ENE method can be reduced to $\mathrm{O}(\mathrm{n})$.

\subsection{Link importance analysis of IoT based on ordered binary decision diagram}

The importance of the network link of the IoT is an evaluation method of the link to the reliability of the IoT. The fragile links in the IoT can be determined by analyzing the link importance. Based on this result, the fragile link can be enhanced. This method not only effectively reduces the network failure probability, but also reduces the loss caused by network failure. At present, the research calculation of the fault probability related measure still lacks an effective analysis method for large scale network analysis. Figuring out which link importance analysis method is practical and efficient is also necessary.

Based on the application of ordered binary decision diagram (OBDD) in network reliability, a link importance evaluation method named BO (Birnbaum OBDD) is proposed. The BO method fully considers the link fault randomness. By analyzing the network reliability of link failures, the Birnbaum measure of the link is calculated to achieve the quantitative calculation of the link importance. The BO method takes the calculation of the network reliability as the key step and combines the efficient OBDD structure with the hash table. By identifying the isomorphic subnets in the calculation, the redundant state and repeated calculation can be reduced to improve the computational efficiency. 
For the IoT with $\mathrm{n}$ links, the Birnbaum measure is defined as follows:

$$
I_{i}^{B}(t)=\frac{\partial F(t)}{\partial F_{i}(t)}, \quad 0 \leq i<n \text {. }
$$

In (8), $F(t)$ indicates the fault distribution function of network $\mathrm{G} ; F_{i}(t)$ suggests the fault distribution function of link $\mathrm{e}_{\mathrm{i}}$. From the relationship between the reliability and the fault distribution function, the Birnbaum measure of link $e_{i}$ satisfies the following formula:

$$
I_{i}^{B}(t)=\frac{\partial R(t)}{\partial R_{i}(t)}, \quad 0 \leq i<n
$$

In (9), $R(t)$ means the reliability function of the network; $R_{i}(t)$ represents the reliability function of the link $\mathrm{e}_{\mathrm{i}}$.

For the IoT network $\mathrm{G}$ with $\mathrm{n}$ links, if its corresponding OBDD structure is $\operatorname{OBDD}(\mathrm{G})$, then the Birnbaum measure of the link $\mathrm{e}_{\mathrm{i}}$ of the network $\mathrm{G}$ can be calculated as follows:

$$
I_{i}^{B}(t)=R E L_{O}\left(\left.O B D D(G)\right|_{x_{j}=1}\right)-R E L_{O}\left(\left.O B D D(G)\right|_{x_{j}=0}\right) .
$$

\section{$4 \quad$ Results}

\subsection{Reliability verification of the IoT}

This paper implements the ENE method in C language and compares it with the method of studying the unreliable node network and the method of studying the edge unreliable network through the experiment. The former comparison method uses the variable order optimization method of Singhal to compare the experiment. The latter one uses the heuristic edge order optimization method of Le. Several benchmark networks are tested in the experiment, and the test results are recorded in Table 1. These networks used in testing are often used as benchmark test networks in the literature for verifying and testing the correctness and efficiency of reliability algorithm.

In Table 1, the computing time used to calculate the reliability of nine IoT networks is recorded. The table is divided into two parts, which corresponds to the two contrast experiment results in the contrast experiment. The left half of the table is the comparison experiment result of the unreliable node calculation method. In the experiment, all the nodes have the same work probability value of 0.9 . The right half part of the table is the comparison realization results of the unreliable edge calculation method. In the experiment, all edges have the same working probability of 0.9 . The reliability of these test networks is verified by the reliability analysis tool. The data recorded in the table marked "ENE calculation time" and "comparison method calculation time" are the average computing time of the corresponding method. In the exper- 
iment, after each method runs 20 times, the average computing time is recorded as the time overhead in the table. The execution time more than one hour is marked as "-" in the table.

In the contrast experiment of unreliable nodes, from the data from the left half of the table, networks $1-4$ and network 7 can be seen. These networks are small in scale and simple in topology, and there is little difference between the average operation time of the ENE method and the contrast method (Singhal method). However, for networks 6-8-9, the scale is relatively large. The ENE method is obviously faster than the Singhal method. Particularly with network 6, the Singhal method has more than 1 hour of operation time, whereas the ENE method only takes 2.14083 seconds. The experimental results show that the larger the network size is, the faster the ENE method is compared with the Singhal method.

From the experimental data of the right half of Table 1, we can see that the average computing time of the ENE method and the contrast method (Le method) has little difference in the calculation time of the reliability of the unreliable edge network. For networks $1-5$ and network 7 , the network size is smaller, and the correlation between the nodes is less. The operation efficiency of the comparison method - Le method is slightly better than that of the ENE method. However, for networks 6-8-9, the scale is large, the proportion of the associated edges is relatively large, and network 8 is obvious. Because the Le method's edge order optimization process increases the computational complexity, the operation time of the ENE method is better than that of the Le method. The experimental results show that in a large network and complex edge structure, ENE method is faster than the Le method.

Table 1. Algorithm running time comparison

\begin{tabular}{|c|c|c|c|c|c|c|}
\hline \multirow{2}{*}{$\begin{array}{c}\text { Network } \\
\text { number }\end{array}$} & $\begin{array}{c}\text { Unreliable node comparison experiment } \\
\text { (node work probability of 0.9) }\end{array}$ & \multicolumn{2}{|c|}{$\begin{array}{c}\text { Unreliable edge comparison experiment } \\
\text { (edge work probability of 0.9) }\end{array}$} \\
\cline { 2 - 7 } & Reliability & $\begin{array}{c}\text { Comparison } \\
\text { method calcu- } \\
\text { aation time } \\
\text { (seconds) }\end{array}$ & $\begin{array}{c}\text { ENE calcu- } \\
\text { lation time } \\
\text { (seconds) }\end{array}$ & Reliability & $\begin{array}{c}\text { Comparison } \\
\text { method calcu- } \\
\text { lation time } \\
\text { (seconds) }\end{array}$ & $\begin{array}{c}\text { ENE calcula- } \\
\text { tion time } \\
\text { (seconds) }\end{array}$ \\
\hline 1 & 0.764413 & 0.09625 & 0.05022 & 0.995586 & 0.06315 & 0.06453 \\
\hline 2 & 0.742912 & 0.12634 & 0.06132 & 0.994295 & 0.07142 & 0.07025 \\
\hline 3 & 0.784694 & 1.26127 & 0.35257 & 0.997020 & 0.36022 & 0.37532 \\
\hline 4 & 0.747435 & 0.09423 & 0.06549 & 0.987290 & 0.05213 & 0.05121 \\
\hline 5 & 0.448035 & 0.26110 & 0.10132 & 0.883169 & 0.06244 & 0.08542 \\
\hline 6 & 0.066000 & ---- & 2.14183 & 0.304190 & 3.91820 & 3.20265 \\
\hline 7 & 0.691833 & 0.09765 & 0.05613 & 0.971253 & 0.04422 & 0.04621 \\
\hline 8 & 0.609532 & 72.68643 & 4.38168 & 0.964374 & 9.14888 & 6.35073 \\
\hline 9 & 0.707858 & 57.93066 & 5.40461 & 0.975457 & 6.04330 & 5.73701 \\
\hline
\end{tabular}

The two parts of the experiment show that the ENE method is not only applicable to the reliability calculation of the unreliable network of nodes and edges but also has good operating efficiency in the IoT network with large scale and complex internal structure. 
This section assumes that the reliability of each node is $\mathrm{R}_{1}=0.88$, the reliability of the system is $\mathrm{R}_{0}=0.95$, and the effective node coverage of the normal work of the system is $\mathrm{r}=80 \%$ to illustrate the practicability of the optimized model. The correlation diagram between system reliability and node number is shown in Figure 6.

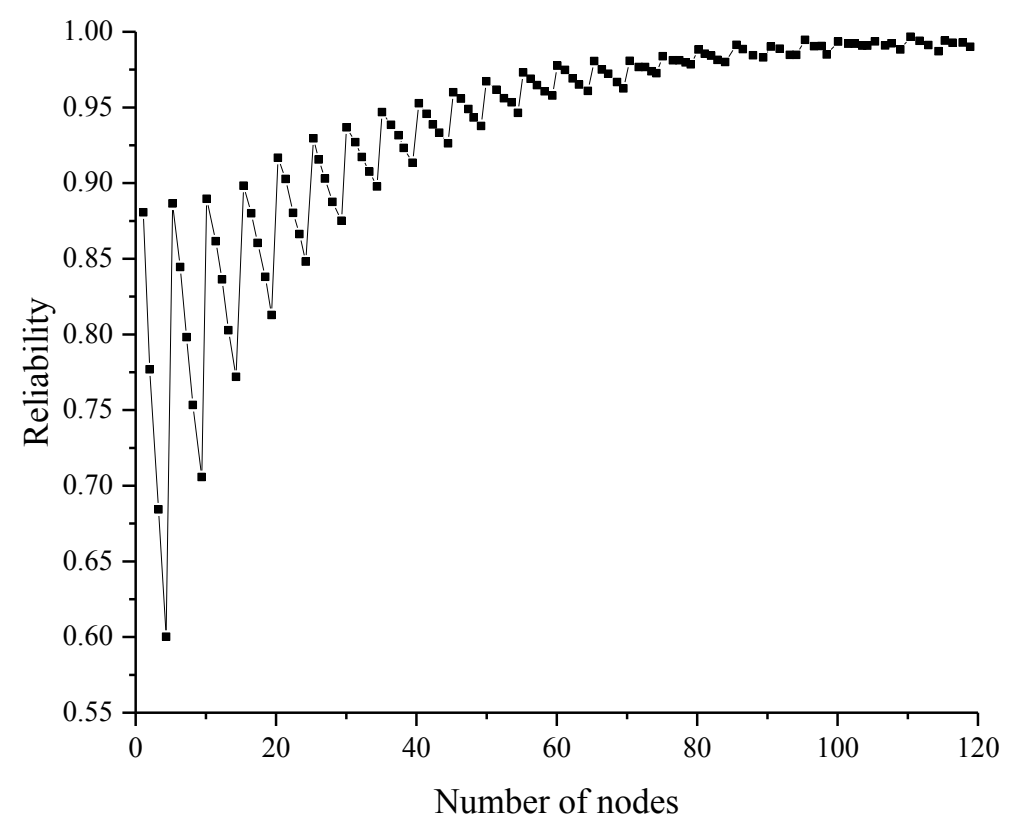

Fig. 6. Correlation diagram between system reliability and node number

As can be seen from Figure 6, the system reliability is not a monotonous number of nodes, thus finding out the number of nodes corresponding to the reliability of the first method is necessary. This example shows that when the IoT system contains 40 nodes, resources are saved the most, and the reliability is 0.9557 , which fulfills the required system reliability of 0.95 .

\subsection{Verification of the importance of the IoT}

The BO algorithm is tested and verified, and the verification code is implemented in $\mathrm{C}$ language. The BDD function library designed by Nielsen is used for reference. In the experimental environment, the hardware running computer is configured as $1.8 \mathrm{GHz}$ frequency $\mathrm{CPU}$ and $512 \mathrm{MB}$ memory, and the software operating system adopts Linux system Fedora13.

The maximum calculation overhead of link importance assessment $\mathrm{BO}$ algorithm is based on the computation of reliability. Therefore, the time cost of computing reliability is used as the key parameter to evaluate the efficiency of the algorithm. In the experiment, the improved binary decision diagram (MBDD) method is used as a ref- 
erence to verify the efficiency of the BO algorithm. The test network is a large number of networks selected from the classic reliability literature. These networks are often used as benchmarking networks to test the correctness and efficiency of the two contrast algorithms. The source nodes and destination nodes are represented by black dots in each network graph. The experimental comparison assumes that the link failure probability of these networks is 0.9 . The result of the run test is listed in Table 2. Comparing the two algorithms in the calculation time of reliability, BO algorithm has high efficiency in computing large scale network, especially when calculating the reliability of No.4 test network and No.6 test network, the time gap between MBDD algorithm and BO algorithm is quite large. In addition, from Table 2, the BO algorithm has a very low overhead when calculating the link importance of the network. For example, for No.6 test network with hundreds of links, the computational cost is only more than 10 seconds.

Table 2. Comparison results between reliability and important link analysis experiment

\begin{tabular}{|c|c|c|c|c|c|}
\hline $\begin{array}{c}\text { Network num- } \\
\text { ber }\end{array}$ & Reliability & $\begin{array}{c}\text { MBDD calcula- } \\
\text { tion reliability } \\
\text { time (seconds) }\end{array}$ & $\begin{array}{c}\text { BO calculation } \\
\text { reliability time } \\
\text { (seconds) }\end{array}$ & $\begin{array}{c}\text { The most im- } \\
\text { portant link }\end{array}$ & $\begin{array}{c}\text { BO calculation } \\
\text { importance } \\
\text { time (seconds) }\end{array}$ \\
\hline 1 & 0.99706 & 0.46173 & 0.00361 & 0 & 0.05219 \\
\hline 2 & 0.99531 & 1.13262 & 0.08769 & 1,2 & 0.73401 \\
\hline 3 & 0.90358 & 0.96726 & 0.06543 & 2,3 & 0.82931 \\
\hline 4 & 0.96347 & 137.042 & 0.38056 & 1 & 5.41832 \\
\hline 5 & 0.78348 & 12.64221 & 0.13412 & 1 & 2.4334 \\
\hline 6 & 0.30332 & 732.84412 & 0.62818 & 1 & 11.4319 \\
\hline
\end{tabular}

\section{Conclusion}

The IoT two-terminal network connectivity reliability is studied under the known network topology. First, the ENE method is proposed based on the OBDD analysis method, which is used to study the two-terminal reliability evaluation of the IoT network with unreliable nodes and edges. Experimental verification of nine benchmark networks shows that the operating efficiency of ENE method is better than that of other methods for large networks. The Birnbaum is used to measure the importance of the IoT link and BO algorithm based on the OBDD analysis method is proposed. The value of the Birnbaum measure is obtained by the reliability calculation to evaluate the link importance of the network. Through experimental comparison, the ability of the BO method to accurately and effectively analyze the importance of network links is verified. Moreover, compared with the contrast method, the BO method can also calculate the quantized value of the importance of the link, so that the order of the importance of any link can be compared, indicating that the BO algorithm is better. The method proposed here has practical value for promoting the reliability research of IoT and guiding the design and development of IOT system. 


\section{$6 \quad$ References}

[1] Suárez, A. D., Parra, O. J. S., \& Forero, J. H. D. Design of an Elevator Monitoring Application using Internet of Things. International Journal of Applied Engineering Research, 2018, vol. 13(6), pp. 4195-4202.

[2] Pan, X. The Design and Reliability Analysis of Elevator Monitoring System Based on the Internet of Things. International Journal of Smart Home, 2016, vol. 10(12), pp. 183-192 https://doi.org/10.14257/ijsh.2016.10.12.17

[3] Li, L. Gu, T. Chang, L. Xu, Z. Liu, Y. Ciphertext-Policy Attribute-Based Encryption Based on an Ordered Binary Decision Diagram. IEEE Access, 2017, vol. 5(99), pp. 1137 1145 https://doi.org/10.1109/ACCESS.2017.2651904

[4] Bollig, B. On the Minimization of (Complete) Ordered Binary Decision Diagrams. Theory of Computing Systems, 2016, vol. 59(3), pp. 532-559 https://doi.org/10.1007/s00224-0159657-x

[5] Brosenne, H. Homeister, M. Waack, S. Nondeterministic Ordered Binary Decision Diagrams with Repeated Tests and Various Modes of Acceptance. Information Processing Letters, 2017, vol. 98(1), pp. 6-10 https://doi.org/10.1016/j.ipl.2005.11.011

[6] Gubbi, J. Buyya, R. Marusic, S. Palaniswami, M. Internet of Things (IoT): A Vision, Architectural Elements, and Future Directions. Future Generation Computer Systems, 2013, vol. 29(7), pp. 1645-1660 https://doi.org/10.1016/j.future.2013.01.010

[7] Perera, C. Zaslavsky, A. Christen, P. Georgakopoulos, D. Context Aware Computing for The Internet of Things: A Survey. IEEE Communications Surveys \& Tutorials, 2013, vol. 16(1), pp. 414-454 https://doi.org/10.1109/SURV.2013.042313.00197

[8] Al-Fuqaha, A. Guizani, M. Mohammad,i M. Aledhari, M. Ayyash, M. Internet of Things: A Survey on Enabling Technologies, Protocols, and Applications. IEEE Communications Surveys \& Tutorials, 2015, vol. 17(4), pp. 2347-2376 https://doi.org/10.1109/COMST.20 $\underline{15.2444095}$

\section{$7 \quad$ Author}

Wei Guan, is associate professor at the Department of Computer Information Engineering, Guangdong Polytechnic of Water Resources and Electric Engineering, Guangzhou 510635, China (guanwei1973@21cn.com). His research interests include Internet of Things.

Article submitted 03 July 2018. Final acceptance 28 July 2018. Final version published as submitted by the authors. 